\title{
Deducing nanopore structure and growth mechanisms in porogen-templated silsesquioxane thin films
}

\author{
Hua-Gen Peng, Richard S. Vallery, Ming Liu, William E. Frieze, and David W. Gidley ${ }^{\text {a) }}$ \\ Physics Department, University of Michigan, Ann Arbor, Michigan 48109 \\ Jin-Heong Yim \\ Division of Advanced Materials Engineering, Kongju National University, 182, Sinkwandong, \\ Kongju City, Chungnam, 314-701, Korea \\ Hyun-Dam Jeong and Jongmin Kim \\ Materials Laboratory, Samsung Advanced Institute of Technology, San 14-1, Nongseo-ri, Kiheung-eup, \\ Yongin-shi, Kyungki-do, 449-712, Korea
}

(Received 28 March 2005; accepted 23 August 2005; published online 10 October 2005)

\begin{abstract}
Adjusting the functional group of a porogen is found to have a tremendous effect on the pore structre of porous low dielectric constant films with silsesquioxane as the matrix precursor. The pore size and interconnection length measured by positronium annihilation lifetime spectroscopy can be used to deduce the pore shape and its evolution with porosity from templates of isolated porogen molecules through film percolation. Inert, self-linkable, and amphiphilic porogens are demonstrated to randomly aggregate three-dimensionally, linearly polymerize, and form micelles, respectively. (C) 2005 American Institute of Physics. [DOI: 10.1063/1.2103402]
\end{abstract}

Porous materials find extensive applications as catalysts, adsorbents, membranes, and recently, as low dielectric constant (low- $k$ ) materials. ${ }^{1}$ The method by which certain parts of the original material structure are selectively removed is widely used to generate a variety of mesoporous materials. ${ }^{2,3}$ The resulting pore structure (size, shape, and interconnectivity) of these materials plays a dominant role in determining the mechanical and electrical properties, as well as their gas and chemical transport capability. In low- $k$ applications, introducing porosity into the interlayer dielectric will reduce the line-to-line resistance-capacitance delay of large-scale integrated circuits. The processing vulnerability of porous structures, however, presents a great challenge for the integration of such materials, ${ }^{4}$ and thus great efforts are made to generate pore structures compatible with microchip fabrication processing.

In typical low- $k$ film pore generation schemes, an unstable pore generator (porogen), such as an organic compound, is mixed with a low- $k$ matrix precursor in solution and then spin cast to form a nanocomposite film. Thermal and/or ultraviolet treatment cures the matrix and degrades the porogen to leave pores behind. ${ }^{5}$ The exact mechanisms of the pore formation process are not well understood, e.g., how pore size, distribution, and interconnectivity are determined from the degradation of the porogen domains. Ideally, the porogen molecules would be separated from each other in the low- $k$ matrix, thus providing a template for isolated nanopores the size of the porogen molecule. However, with increasing porogen concentration, the porogen-porogen interaction is enhanced relative to that of the porogen-matrix, leading to greater aggregation of the porogen domains. Thus, lowering $k$ by increasing porosity reaches a limit when the pore structure becomes unacceptably susceptible to integration damage.

\footnotetext{
${ }^{a)}$ Electronic mail: gidley@umich.edu
}

In this letter, we prepare porous silsesquioxane (SSQ) low- $k$ films with three porogens of different functional groups. Positronium annihilation lifetime spectroscopy (PALS) is then utilized to deduce the mean-free path (MFP), and interconnection length, ${ }^{6} \mathrm{~L}_{\text {int }}$, of the pores. As the porogen content of a film is increased, the evolution of these pore metrics provides critical input as to how the porogen molecules aggregate, eventually leading to film percolation (a porous network throughout the film).

The detailed experimental procedure for making the porous SSQ films has been published elsewhere. ${ }^{7}$ A modified cyclic SSQ (mCSSQ) with controlled $\mathrm{Si}-\mathrm{OH}$ content is used as the low- $k$ matrix precursor. ${ }^{8}$ Solutions of the MCSSQ precursor and porogen of varying weight loadings [using propylene glycol methyl ether acetate (PGMEA), as the solvent] were spin coated onto a silicon substrate. Porous films were then made after successive hot-plate bakes at 100 and $250{ }^{\circ} \mathrm{C}$ followed by final curing in a vacuum furnace held at $420^{\circ} \mathrm{C}$. The film thickness (nominally 1 micron) and refractive index are measured by a surface profiler and a prism coupler. The porogen-induced porosities $(p)$ of the films are calculated from the Lorentz-Lorenz equation and the measured refractive index of a matrix film which defines $p=0$.

The model porogens, whose chemical structures are shown in Fig. 1, consist of two modified cyclodextrins (tCD and $\mathrm{sCD})$ and one calix-arene $(\mathrm{CA}[6])$ that have been demonstrated to be promising supramolecular pore generators. ${ }^{7}$ The CDs are cyclic oligosaccharides consisting of six glucopyranose units that are joined together by $\alpha(1 \rightarrow 4)$ linkages. $\mathrm{CA}[6]$ is a six-member arene ring with acetoxyl and tert-butyl groups. All of the porogen molecules assume a truncated cone structure. For the CDs, the rigid core has a maximum diameter of $\sim 1.5 \mathrm{~nm}$ and a height of $\sim 0.8 \mathrm{~nm}{ }^{7}$ The corresponding dimensions of $\mathrm{CA}[6]$ are slightly larger at $1.6 \mathrm{~nm}$ and $0.9 \mathrm{~nm}^{9}$

Characterization of nanopore structure in thin, amorphous, films is very challenging. Relatively few techniques have emerged to determine pore size, such as PALS, ellipso- 


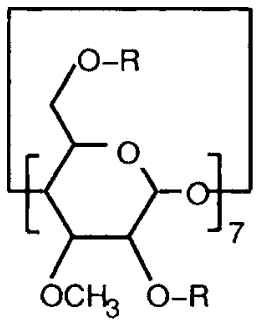

(a)

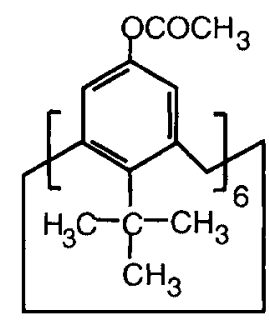

(b)
FIG. 1. Chemical structures of the porogen molecules: tCD, Heptakis (2,3,6-tri-O-methyl)- $\beta$-cyclodextrin, (a) with $R=\mathrm{CH}_{3} ; \quad s \mathrm{SD}$, Heptakis(3-O-methyl-tetradecakis-2, 5-di-O-[3-(trimethoxysilyl)propyl]- $\beta$ cyclodextrin, (a) with $R=$ (trimethoxysilyl)propyl; and CA[6] 5,11,17,23,29,35-hexa-tert-butyl-37,38,39,40,41,42-hexa-acetoxyl calix arene, (b).

metric porosimetry, small-angle x-ray/neutron scattering, and $\mathrm{x}$-ray porosimetry. ${ }^{10} \mathrm{It}$ is even more difficult to deduce pore shape and interconnectivity and their evolution with porosity. Beam-based PALS provides direct information on the MFP (an average size measure of a pore of any shape, volume $V$, and surface area $S$ where the $\mathrm{MFP}=4 \mathrm{~V} / \mathrm{S}$ ) and $L_{\text {int }}$, the length scale over which pores are interconnected to each other. ${ }^{6}$ In this technique, a focused monoenergetic beam of positrons is implanted and forms positronium (Ps, the electron-positron bound state) by electron capture. Ps inherently localizes in the pores where its intrinsic vacuum lifetime of $140 \mathrm{~ns}$ is reduced by annihilation with bound electrons during collisions with the pore surfaces. The collisionally reduced Ps lifetime is well calibrated with the void size. ${ }^{11}$ The detection of Ps annihilating in the vacuum with $140 \mathrm{~ns}$ is the telltale sign of Ps diffusing through an interconnected porous network and escaping into vacuum, after as many as a million collisions with the pore surfaces.

Details of PALS methodology have been presented elsewhere. ${ }^{6}$ Briefly, lifetime spectra are acquired at positron beam energies ranging from $0.55 \mathrm{keV}$ to $5.0 \mathrm{keV}$ which provide mean positron implantation depths $(\bar{h})$ of roughly 10 to $400 \mathrm{~nm}$. These spectra require one very short positron lifetime and four Ps lifetimes for adequate fitting. The shortest two Ps lifetimes are 2.5 and $7 \mathrm{~ns}$ and correspond to $0.5-1.0$ $\mathrm{nm}$ micropores inherent to the mCSSQ matrix (verified by analyzing a matrix film with no porogen). The longest component corresponds to the 140 ns vacuum annihilation lifetime of Ps. The nonzero intensity of this component (after backscattering subtraction) is attributed to Ps that has diffused out of the film through interconnected mesopores. The fourth, intermediate lifetime component ranging from 12-60 ns corresponds to Ps annihilating in the porogen-induced mesopores. The intensity of this Ps component is zero for the matrix, and increases with porogen content (at the expense of the micropore Ps intensity), but then decreases at higher porosities as Ps escape into vacuum shifts its intensity into the 140 ns component. The fraction of Ps escaping from the mesopore, $F_{\text {esc }}$, is readily calculated versus $\bar{h}$ and then a simple diffusion model is used to estimate the Ps diffusion length for each film porosity. We take this diffusion length as an average measure of $L_{\text {int }}{ }^{6}$ The MFP of the pores is determined $^{11}$ from the fourth Ps lifetime. However, for samples with nonzero $F_{\text {esc }}$, films with a thin silica sealing layer to block Ps escape are analyzed to ensure correct mea-

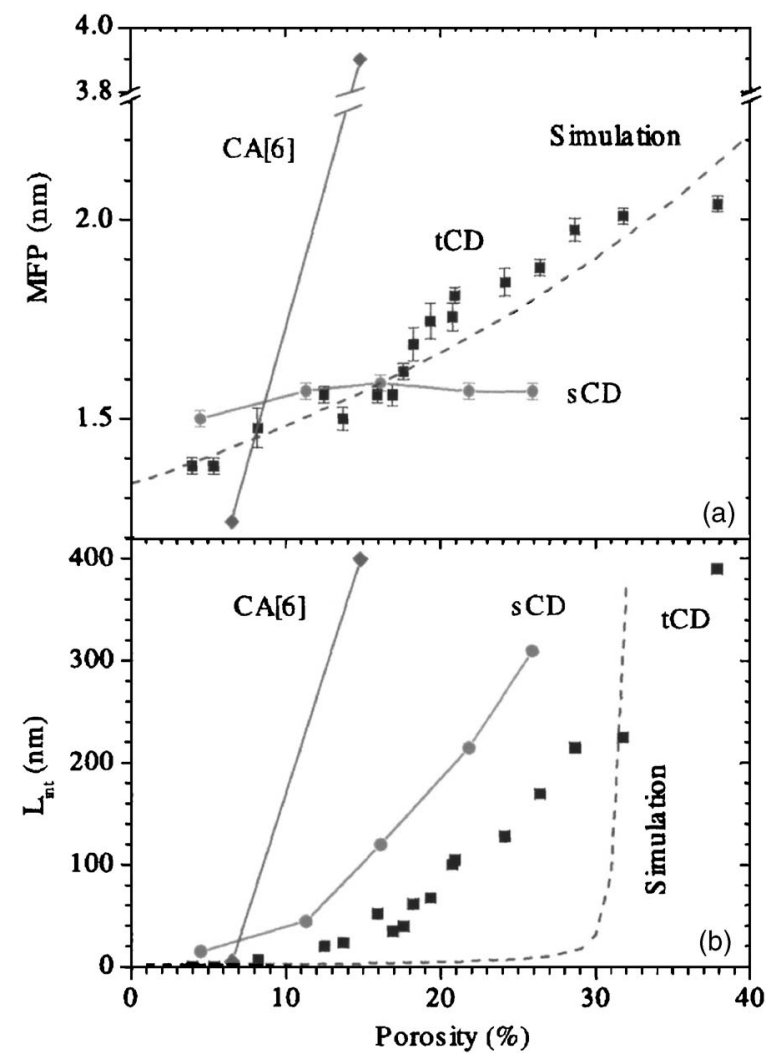

FIG. 2. MFP (a) and $L_{\text {int }}$ (b) vs porosity for films made with different porogens. Dashed curves are derived from a simulation in which cubic pores of side length $=2.0 \mathrm{~nm}$ are randomly placed in a cubic lattice.

surement of the Ps lifetime inside the porogen-induced pores. $^{12}$

In Figs. 2(a) and 2(b), we present the deduced MFP and $L_{\text {int }}$ versus porosity. At all but the lowest porosities, we favor a cylindrical pore model as physically more reasonable given the nonzero values of $L_{\text {int }}$. However, the resulting MFP $(4 \mathrm{~V} / \mathrm{S})$, which is the cross-sectional diameter in a long cylinder, is only about $20 \%$ larger than the MFP deduced using a spherical pore model. (We are not a priori implying the pores are cylindrical.) The trends in the Fig. 2 suggest three distinct growth modes of porogen aggregation with concentration.

The sCD system clearly forms pores with constant MFP throughout the porosity range, despite the fact that $L_{\text {int }}$ increases rapidly with porosity. Even at low $4 \%$ porosity the $1.5 \mathrm{~nm}$ MFP pores have interconnection length ten times this value. The pores are clearly not isolated, but instead display the effect of strong linear polymerization of the SCD molecules through polycondensation. Apparently, water residue in the solvent/precursor or produced by condensation of the mCSSQ precursor promotes fast hydrolysis of the trimethoxylsilyl end groups, through which $\mathrm{Si}-\mathrm{O}-\mathrm{Si}$ bonds are formed between neighboring SCD molecules. The porogen domains are driven by strong covalent bonding to be cylindrical and increased porogen concentration simply lengthens them. The MFP of 1.5-1.6 nm corresponds nicely to the sCD ring diameter which becomes the cylindrical diameter of the porogen template as end-group bonding polymerizes the sCD rings axially.

For the tCD porogen, Fig. 2(b) indicates that for $p$ $\leqslant 5 \%$ the tCD-induced pores are isolated since $L_{\text {int }} \approx 0$. For $p \geqslant 8 \%$, pores start to interconnect and the size of pores con- 
tinuously increases. The MFP of pores at low porosity, 1.3$1.4 \mathrm{~nm}$, is larger than the calculated $4 \mathrm{~V} / \mathrm{S}$ for a single $\mathrm{tCD}$ molecule, which is $\sim 0.8 \mathrm{~nm}$. However, a long cylinder is not a good model for isolated pores, so we should instead use a spherical pore model to convert the fitted Ps lifetime of $16 \mathrm{~ns}$ into a spherical pore diameter of $1.65 \mathrm{~nm}(\mathrm{MFP}=1.1 \mathrm{~nm})$. This size is consistent with two $(1.0 \mathrm{~nm})$ or three $(1.15 \mathrm{~nm})$ back-to-back tCD molecules, suggesting that $\mathrm{tCD}$ molecules are predominantly not singly dispersed even though no chemical bonding exists between them. For molecules such as $t C D$, there are simulations and experiments demonstrating such self-assembly capability. ${ }^{13}$ The preferred organization is neighboring tCD molecules align their bottom planes (or upper planes) parallel to each other through van der Waals interactions. Our PALS measurements corroborate these findings. The minimum porogen domains appear to be at least two tCD molecules. The MFP steadily increases with porosity well beyond that of the cylindrical pores in the SCD system. This must result from some degree of three dimensional (3D) aggregation of these small porogen domains. For comparison, we have plotted the simulated growth in the MFP of cubic pores randomly placed in a cubic lattice. ${ }^{14}$ The tCD pore MFP growth is consistent with random aggregation in three dimensions. However, $L_{\text {int }}$ is extracted from the very same simulation and the measured $L_{\text {int }}$ for tCD increases much sooner than expected from simple 3D random growth. Evidently, the weak, but favorable, porogen-porogen interactions can lead to enhanced pore interconnection length prior to the percolation limit in the simulated lattice filled with truly random pores.

The CA[6] system is quite different. In Fig. 1(b), the upper plane of $\mathrm{CA}[6]$ molecules are hydrophilic, while the bottom plane is hydrophobic. Only the upper plane can form hydrogen bonds with hydroxyl groups from the SSQ matrix and, thus CA[6] may act as a supramolecular "surfactant" in the SSQ precursor solution with a hydrophilic solvent PGMEA. At low porosity of 7\%, Fig. 2 indicates isolated pores $\left(L_{\text {int }}=0\right)$ that are consistent with the size of the CA[6] molecule. The fitted $12.5 \mathrm{~ns}$ Ps lifetime should again be interpreted using a spherical pore model to yield a diameter of $1.45 \mathrm{~nm}$ with a MFP just below $1.0 \mathrm{~nm}$, in agreement with the calculated $4 \mathrm{~V} / \mathrm{S}=0.9 \mathrm{~nm}$ for a single molecule). In stark contrast, at $p=14 \%$, the pore interconnection length of 400 $\mathrm{nm}$ indicates film percolation accompanied with explosive growth in pore size to $3.9 \mathrm{~nm}$. This pore size far exceeds the dimension of random 3D cluster growth of the porogen domain, as suggested for tCD. Percolation occurring at such low porosity as $14 \%$ strongly suggests that the pores are highly elongated. A simple explanation of cylindrical micelle formation appears a very likely interpretation for the sudden onset of such large and interconnected pores. Quite clearly a critical concentration for micelle formation exists between $7 \%$ and $14 \%$ porosity. A study with much finer concentration increments is warranted to observe the evolution of this special mode of porogen aggregation through its threshold for micelle formation.
In conclusion, simultaneously resolving the evolution with porosity of the mean size $(4 \mathrm{~V} / \mathrm{S})$ and extent $\left(L_{\text {int }}\right)$ of porogen-induced pores over the range from isolated porogen molecules to large self-assembled clusters provides powerful clues to deduce fundamental pore structure and growth. The sCD porogen system demonstrates 1D linear growth driven by covalent bonding to form ever longer pores of constant cross section (constant MFP with increasing $L_{\text {int }}$ ). The sudden jump in both MFP and $L_{\text {int }}$ in CA[6] is consistent with surfactant driven cylindrical self-assembly above a critical porogen concentration [below which the $\mathrm{CA}[6]$ domains are simply isolated single molecules]. The tCD system displays elements of short-range 1D growth to form two to three molecule clusters that give way to a more dominant, random 3D growth with porosity, all driven by weaker van der Waals interactions. However weak these porogen interactions are, the growth in $L_{\text {int }}$ is much more strongly affected than the MFP compared to truly random (zero-interaction) 3D growth. The discovery of these three distinctly different domain/pore growth modes demonstrates the versatility and capability of depth-profiled PALS to characterize pore structure. As such, these PALS results appear to be quite unique in probing pore structure evolution in the prepercolation regime.

This work is supported by the Samsung Advanced Institute of Technology. Associated research at Michigan is funded by the National Science Foundation (No. ECS0100009) and the Low- $K$ Dielectric Program at International Sematech.

${ }^{1}$ F. Schüth, K. S. W. Sing, and J. Weitkamp, Handbook of Porous Solids (Wiley-VCH, Weinheim, 2002).

${ }^{2}$ C. T. Kresge, M. E. Leonowicz, W. J. Roth, J. C. Vartuli, and J. S. Beck, Nature (London) 359, 710 (1992).

${ }^{3}$ R. A. Pai, R. Humayun, M. T. Schulberg, A. Sengupta, J. N. Sun, and J. J. Watkins, Science 303, 507 (2004).

${ }^{4}$ M. Fayolle, G. Passemard, O. Louveau, F. Fusalba, and J. Cluzel, Microelectron. Eng. 70, 255 (2003)

${ }^{5}$ J. L. Hedrick, R. D. Miller, C. J. Hawker, K. R. Carter, W. Volksen, D. Y. Yoon, and M. Trollsas, Adv. Mater. (Weinheim, Ger.) 10, 1049 (1998).

${ }^{6}$ J. N. Sun, D. W. Gidley, Y. F. Hu, W. E. Frieze, and S. Yang, Mater. Res. Soc. Symp. Proc. 726, Q10.5 (2002).

${ }^{7}$ J. H. Yim, J. B. Seon, T. D. Jeong, L. Y. S. Pu, M. R. Baklanov, and D. W. Gidley, Adv. Funct. Mater. 14, 277 (2004).

${ }^{8}$ J. H. Yim, Y. Y. Lyu, H. D. Jeong, S. K. Mah, J. Hyeon-Lee, J. H. Hahn, G. S. Kim, S. Chang, and J. G. Park, J. Appl. Polym. Sci. 90, 626 (2003).

${ }^{9}$ C. D. Gutsche, B. Dhawan, K. H. No, and R. Muthukrishnan, J. Am. Chem. Soc. 103, 3782 (1981), and the dimensions of the molecule are based on the semi-empirical quantum mechanic theory (PM3): J. J. P. Stewart, J. Comput. Chem. 10, 209 (1989).

${ }^{10}$ K. Maex, M. R. Baklanov, D. Shamiryan, F. Iacopi, S. H. Brongersma, and Z. S. Yanovitskaya, J. Appl. Phys. 93, 8793 (2003), and references therein.

${ }^{11}$ T. L. Dull, W. E. Frieze, D. W. Gidley, J. N. Sun, and A. F. Yee, J. Phys. Chem. B 105, 4657 (2001), and references therein.

${ }^{12}$ D. W. Gidley, W. E. Frieze, T. L. Dull, A. F. Yee, E. T. Ryan, and H. M. Ho, Phys. Rev. B 60, R5157 (1999).

${ }^{13}$ S. Polarz, B. Smarsly, L. Bronstein, and M. Antonietti, Angew. Chem., Int. Ed. 40, 4417 (2001).

${ }^{14}$ J. Hoshen and R. Kopelman, Phys. Rev. B 14, 3438 (1976). 